\title{
Structural insights into bacterial lipoprotein biosynthesis
}

\section{Cameron L. Noland, Michele Kattke, Susan Gloor, Jingyu Diao, Homer Pantua, Sharookh}

Kapadia, and Jeremy M. Murray

The outer membrane of gram-negative bacteria contains a diverse array of lipoproteins that are essential for cell viability. Lipoprotein biosynthesis requires the sequential activity of three enzymes that are embedded in the cytoplasmic membrane. First, prolipoprotein

diacylglyceryl:phosphatidylglycerol transferase (Lgt) recognizes a conserved lipobox motif within the prolipoprotein signal sequence and catalyzes the addition of diacylglycerol to an invariant cysteine. The signal sequence is then cleaved by signal peptidase II (LspA), generating a mature N-terminus at the conserved cysteine. In the final step of lipoprotein biosynthesis, prolipoprotein $\mathrm{N}$-acyltransferase (Lnt) catalyzes the transfer of the $s n$-1-acyl chain of phosphatidylethanolamine to this N-terminal cysteine, generating a mature, triacylated lipoprotein. Recent structural studies of Lgt and LspA have yielded significant mechanistic insights into this essential biosynthetic pathway. However, the structure and mechanism of Lnt has remained elusive. We have determined the atomic structure of Escherichia coli Lnt in its apo state at $2.5 \AA$ resolution. The structure reveals a monomeric 8-transmembrane helix fold that supports a periplasmic carbon-nitrogen hydrolase domain containing a canonical Cys-Glu-Lys catalytic triad. Through complementary molecular dynamics and biochemical approaches, we provide novel mechanistic insight into lipoprotein $\mathrm{N}$-acylation in gram-negative bacteria.

$\begin{array}{lll}\text { Cameron Noland } & \text { USA } & \text { Genentech } \\ \text { Michele Kattke } & \text { USA } & \text { UCLA } \\ \text { Susan Gloor } & \text { USA } & \text { Genentech } \\ \text { Jingyu Diao } & \text { USA } & \text { Genentech } \\ \text { Homer Pantua } & \text { USA } & \text { Genentech } \\ \text { Sharookh Kapadia } & \text { USA } & \text { Genentech } \\ \text { Jeremy Murray } & \text { USA } & \text { Genentech }\end{array}$

